Slavica

bruxellensia

\section{Slavica bruxellensia}

Revue polyphonique de littérature, culture et histoire

slaves

$7 \mid 2011$

Les Slaves, le Midi et l'Orient

\title{
Milostný Dopis Klínovým Písmem / Lettre d'amour en cunéiforme de Tomáš Zmeškal (extraits)
}

Traduction du tchèque

\section{Sarah Flock}

\section{(2) OpenEdition}

\section{Journals}

Édition électronique

URL : http://journals.openedition.org/slavica/899

DOI : $10.4000 /$ slavica.899

ISSN : 2034-6395

Éditeur

Université libre de Bruxelles - ULB

Référence électronique

Sarah Flock, " Milostný Dopis Klínovým Písmem / Lettre d'amour en cunéiforme de Tomáš Zmeškal (extraits) ", Slavica bruxellensia [En ligne], 7| 2011, mis en ligne le 15 octobre 2011, consulté le 01 mai 2019. URL : http://journals.openedition.org/slavica/899 ; DOI : 10.4000/slavica.899

Ce document a été généré automatiquement le 1 mai 2019.

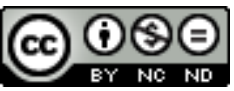

Les contenus de Slavica bruxellensia sont mis à disposition selon les termes de la Licence Creative Commons Attribution - Pas d'Utilisation Commerciale - Pas de Modification 3.0 France. 


\section{Milostný Dopis Klínovým Písmem / Lettre d'amour en cunéiforme de Tomáš Zmeškal (extraits)}

Traduction du tchèque

Sarah Flock

\section{Présentation}

1 Né en 1966 à Prague, Tomáš Zmeškal est d'origine congolaise et tchèque. Après avoir étudié la langue et la littérature anglaises et avoir vécu à Londres pendant plus de dix ans, il est aujourd'hui professeur d'anglais dans un lycée pragois. A côté de son activité pédagogique, il est surtout l'auteur de deux romans publiés aux éditions Torst : Milostný dopis klínovým písmem (Lettre d'amour en cunéiforme, 2008) et Životopis černobilého jehnět (Biographie d'un agneau noir et blanc, 2009). Grâce à son premier livre, Zmeškal reçoit le prix littéraire Josef Škvorecký et devient du jour au lendemain un des auteurs tchèques contemporains les plus lus, comparé par la critique à Josef Škvorecký, Bohumil Hrabal ou Salman Rushdie.

2 Lettre d'amour en cunéiforme est l'histoire d'un triangle amoureux entre deux hommes et une femme. L'intrigue, centrée sur le couple formé par Josef et Květa,débute peu avant la fin de la seconde guerre mondiale et prend fin au début des années 1990. Zmeškal n'a toutefois pas la velléité d'esquisser une fresque historique. Il cherche, en racontant les destins personnels des membres de la famille de Josef et Květa, à refléter la complexité de la société tchèque.

3 Le roman, polyphonique dans sa composition et postmoderne par sa fragmentation, compte vingt-deux chapitres. Chacun d'eux est rédigé dans un style différent en fonction du personnage qui est impliqué dans le récit. A l'instar de Geoffrey Chaucer, Zmeškal introduit subtilement la voix du personnage, devenu alors narrateur, dans le récit. Aussi, y a-t-il dans l'écriture de Zmeškal un réel travail sur la langue. Plus loin, l'histoire, flirtant tour à tour avec l'histoire des mythes, la science-fiction, le grotesque et le tragique, se 
déploie aussi au travers de lettres ou de visions.

\section{Traduction}

\section{Chapitre 8 : Première vision de l'éternité - la Perse}

4 Le docteur Antonin Lukavsky n'était pas vraiment satisfait par l'explication d'un des noms, en particulier celui d'Hitler, que s'était choisi le confiseur Marek Svoboda. Il y avait ici bien sûr des tendances paranoïdes évidentes dans ses relations avec les femmes et avec la société humaine en général. Par ailleurs, il y transparaissait un certain avancement monstrueux de la science, qui était seulement enprisonné dans l'imagination du patient, qui techniquement n'était pas réalisable et qui, à coup sûr, ne pourrait pas être réalisable même dans le futur, et la seule chose qui ressortait de tout cela, c'était l'inclinaison du patient pour les récits extraordinaires. Le docteur Lukavsky réfléchit aussi un instant à la motivation que pouvait avoir son patient pour simuler un désordre psychique. Il manquait cependant un mobile évident. Un changement survint pourtant dans le service. La qualité des plats, du reste habituellement médiocre, s'était rapidement améliorée avec son arrivée. Le confiseur Marek Svoboda sympathisa vite avec le cuisinier de l'hôpital et, en plus de sentir bon, les repas étaient maintenant inhabituellement savoureux et Antonin dut bien remarquer que les patients, le personnel et même lui commencèrent à se délecter de la nourriture servie à la cantine. En ce qui concerne cependant Marek Svoboda, il était évident, après quelques semaines, qu'il n'était plus nécessaire qu'il reste dans le service, il fut renvoyé à la maison et ne devait plus passer qu'une fois par mois au cabinet d'Antonin. Ils parlaient de tout et de rien et Antonin se rendit compte qu'il aimait parler avec lui. Sa rectitude était revigorante. Il leur arriva plusieurs fois de se disputer à propos du sens de la médecine. "Quelle en est tout compte fait le sens ?" demandait Monsieur Svoboda. "La santé !" rétorquait le docteur Lukavsky. "Trouve-t-on les causes de chaque maladie et peut-on toutes les guérir ?" continuait Marek. "C'est un problème abstrait qui ne me concerne pas car je ne vivrais pas assez longtemps pour le savoir." répondait Antonin. "Que vient-il après ?" demandait Marek. "Après se révèle sa vraie essence qui est le désir d'immortalité. Ça, j'en suis presque certain, mon frère." dit-il avec conviction. Et ensuite, il lui dit : "J'ai eu deux visions à ce sujet, deux visions d'éternité." Aujourd'hui, je te raconte celle qui s'est déroulée loin d'ici et dans le passé. Le prochain jeudi, quand je devrai revenir ici, je te raconterai la seconde qui se déroule ici mais dans le futur." Il commença: "Nous marchions dans les montagnes. Moi, ma femme et mon jeune frère, quelques parents, des porteurs, des auxilliaires et des serviteurs. J'avais déjà cessé de me demander si nous étions des réfugiés ou des commerçants. D’ailleurs, la différence ne semblait pas être tellement grande. Nous étions entourés par de hautes montagnes. Et quand j'ai dit que nous marchions dans les montagnes, j'aurais dû plutôt dire que nous nous efforcions de les éviter. Marcher dans les montagnes ne signifie pas marcher à travers les montagnes. Nous tentions de ramper, tels des serpents malins, à travers les cols et les gorges, de soudoyer les gardes, de payer le guide, d'éviter les douanes, nous tentions d'échapper à notre destin naturel. Nous tentions l'exploit téméraire de survivre. Nous écoulions différentes sortes de marchandises, faisions entrer en contrebande différentes sortes de marchandises, nous vendions et achetions tout ce qu'il était possible de vendre et d'acheter et, pourtant, nous n'avions les moyens que de 
sauver la nudité de nos vies. Nous nous dirigions vers la Perse et devions éviter une troupe armée après l'autre. Je voulais être à Tabriz pour le debut du printemps, j'y avais négocié un magasin l'an passé. Nous étions sortis un peu plus tôt cette année, le printemps s'était pourtant transformé d'une jeune fille en bourgeon en une vieille femme dont le visage était variolé. L'hivers n'était pas parti, c'était en fait le contraire.

Le douzième jour de notre voyage, j'ai remarqué que ma femme avait l'air plus tourmentée qu'auparant et qu'elle tenait son cheval seulement au prix d'un grand effort. $\mathrm{Au}$ début, j'ai cru qu'il s'agissait de ses problèmes féminins, que Dieu a, dans sa toute puissance, infligé aux femmes pour les punir de la souffrance qu'elles ont causé aux hommes. Mais ensuite, j'ai entendu sa toux qui craquait comme du bois sec que l'on allume. Ses yeux étaient ternes, ses cheveux bleutés avaient perdu leur éclat et j'ai commencé à avoir peur d'être incapable de la protéger d'Azraël, l'ange de la mort. Je n'ai pas fermé l'œil pendant plusieurs jours, et, quand, pendant une semaine, elle s'est endormie la tête sur mes genoux et que, chaque soir, j'entendais comme sa respiration était sèche, j'ai décidé de rentrer. Mais, je me suis décidé trop tard. Une toujours autre et nouvelle maudite armée d'une autre maudite grande puissance, cette fois venue de cette damnée Europe, poussait devant elle, comme des insectes, une nouvelle vague de fuyards mourants. Le chemin vers la maison en faisant marche arrière par le Nord était désormais impossible car notre Dieu, dont la vue fait naître et mourir les étoiles, nous avait gratifié d'un autre coup : les barbares tentaient à nouveau de dominer nos cols, nos montagnes, notre minéral, la richesse de notre terre et ce qui se cache sous elle, et j'ai seulement prié pour que Dieu, dont le nom est éternel, les gratifie de la même mortification que celle dont il nous avait alors gratifié. Mon jeune frère tâchait de trouver un médecin et un sage, qui nous avaient été conseillés par un ami, marchand de tapis, toutefois lorsque nous sommes arrivés à l'endroit indiqué, nous avons trouvé tout un village vide et marqué par le feu. J'ai parcouru la première, la deuxième, la troisième maison brûlée puis j'ai ordonné que l'on remonte sur les chevaux, bien que mes hommes aient toute une journée de randonnée derrière eux, et, j'ai dû faire cesser leur plainte par quelques coups de fouets. J'ai ordonné qu'on ne perde pas de temps à ensevelir les corps des pauvres car je ne voulais pas partager leur destin. J'ai demandé de charger les fusils et de ne pas offrir de manière totalement gratuite notre vie misérable. Je préférais ne pas regarder ma femme, je suis allé au devant de mes hommes et j'ai demandé à mon frère de ne pas la quitter des yeux et de me dire lorsque l'ange de la mort serait en vue. A midi, nous avons rattrapés d'autres commerçants qui se sont volontier joints à nous. Cela m'a réjouit car je ne connaissais pas trop bien la route. Au moment de la prière, mon frère est venu près de moi et m'a dit que la vie de mon épouse ne tenait qu'à une fine cordelette de soie d'espoir. Tristement, il a ajouté que le fil allait vite se rompre. Un des commerçants qui venaient de se joindre à nous l'a entendu et m'a dit qu'il avait beaucoup appris de son père qui était médecin, et il m'a demandé s'il pouvait examiner ma femme. Je suis allé avec lui auprès d'elle et, après deux jours, je l'ai revue. Elle était plus belle qu'avant et elle était en train de mourir. J'ai laissé le commerçant qui avait proposé de l'examiner avec mon frère, et, je suis allé prier Dieu dont la miséricorde est grande, j'ai prié et je lui ai demandé conseil. Que faire? Nous ne connaissons pas les environs. Au nord, des barbares sanguinaires. Au nord-est, des miliers de réfugiés. Tabriz est loin et si nous nous écartons de la route, des barabares européens fanatiques ou d'autres nous attraperons brutalement. Alors que je terminais une deuxième unité de prière, l'homme qui venait d'examiner ma femme m'a rejoint et m'a dit que si nous ne descendions pas dans la vallée et que mon épouse ne 
commençait pas à se rétablir, elle mourrait dans la semaine. Selon lui, je devais descendre sur le champ dans la vallée si je voulais la sauver. Car Dieu qui pleure sur nos actes m'a puni de mon amour obstiné et excessif pour mon épouse souffrante, je me suis décidé tout de suite. J'ai encore pris conseil auprès d'autres marchands qui connaissaient la région et j'ai aussitôt donné l'ordre de faire un détour par la vallée la plus proche. La vallée devait se trouver à seulement quelques heures de marche rude, nous devions l'atteindre avant la tombée de la nuit. Dans ma tête, je remerciais Dieu qui connaît les désirs enfouis dans nos coeurs et j'ai ordonné le détour. Nous étions quelque part entre l'Arménie et la Perse. J'ai envoyé en éclaireur mon meilleur cavalier sur le meilleur cheval, donc sur le mien. J'ai commandé à mon frère qu'il prennne soin de ma femme comme de la prunelle de ses yeux et nous avons commencé à descendre. Le chemin était broussailleux, rocailleux et n'avait plus été utilisé depuis des années. Seul un ruisseau de montagne le descendait chaque printemps. Le chemin ressemblait à une pelotte démaillée de serpents, j'ai senti en descendant que certains de nos animaux risquaient de se rompre les ligaments. Cela n'est pas arrivé. Les animaux ont été plus chanceux qu'un de mes serviteurs qui est tombé et a roulé jusqu'en bas. Quelques heures plus tard, nous avons enseveli son corps au pied de la montagne. Un autre serviteur a essayé de profiter de mon expédition en pente et a tourné discrètement avec son cheval et quelques autres bêtes. Tout d'abord, il est descendu de son cheval et, à l'aide d'un couteau, a retiré une pierre de son sabot. Cette ruse était trop vieille pour me duper, en plus, il avait un regard louche. La dernière fois que je lui avais parlé, ses yeux étaient restés fixés sur un bouton de ma veste et seul Dieu, dans son infinie compassion, peut ensuite se montrer clément envers quelqu'un en qui je ne peux pas croire. Et donc j'ai attendu à une autre boucle du chemin, et comme il ne venait pas, je suis parti à sa rencontre. Lorsque je l'ai vu et qu'il n'a pas répondu à mon appel et a seulement poussé les animaux plus loin dans la montagne, une balle de mon fusil a ramené son âme indigne près de Dieu qui est le père bienveillant de tous et de tout.

6 Avant le coucher du soleil, nous étions dans la vallée et le premier village était en vue. D'après les maisons que je voyais, il devait y avoir environ deux cents personnes dans le village. Au milieu coulait une rivière morcelée, au dessus de laquelle se courbait un pont dont les piliers étaient si joliment décorés que je n'en avais vu de semblables qu'à Bagdad pendant mon enfance, lorsque je faisais mon premier voyage marchand avec mon père. Mon souci principal était de trouver un médecin. J'en ai trouvé finalement deux dans le village. Au début, aucun d'eux n'a voulu venir car nous étions étrangers et que, d'après leurs coutumes, ils n'examinent pas d'étranger après le coucher du soleil. Je leur ai d'abord proposé de l'argent, le premier des deux l'a pris et est allé soigner ma femme. Le second a seulement haussé les épaules et a voulu rentrer chez lui. Je voulais pourtant être sûr que mon épouse allait vivre, alors j'ai dû d'abord lui casser quelques côtes pour qu'il comprenne que je ne laisserais pas ma fleur la plus chère dans les mains d'un seul guérisseur. Après avoir changé les draps qui l'enveloppait, lui avoir fait boire du thé à base d'herbes médicinales et avoir préparé un onguent, ils se sont aperçus que les personnes qui leur étaient chères et proches avaient toutes été enfermées dans deux pièces d'une auberge que je venais d'occuper avec mes hommes. Je leur ai dit clairement que, si ma femme mourait, elle ne mourrait pas seule. Je n'ai pas eu à m'expliquer plus, le soir j'ai seulement redoublé la garde.

7 Le deuxième matin, alors que je venais de me réveiller d'un rêve dans lequel j'avais d'abord essayé de convaincre et ensuite soudoyer Azraël, l'ange de la mort, pour qu'il échange quelques années de ma propre vie contre la vie de ma femme, j’ai entraperçu au 
cours de ma ronde un des guérisseurs en train de laver le châle de mon épouse dans la rivière sous le pont. Cela m'a fait enrager et mon frère est seulement parvenu à me retenir pour que je ne le frappe pas à mort. C'est ça votre thérapie ? J'étais en rage ! Les familles des guérisseurs m'ont toutefois convaincu par leur cri suppliant et par leur gémissement que l'eau de la rivière, sous le pont joliment construit, possédait des vertus curatives. Je les ai crus dès le jour suivant, quand le visage du guérisseur avait retrouvé une peau de nouveau-né et ne portait plus la trace de mes poings et de mes bottes.

Avant d'aller dormir, je suis encore entré en silence dans la chambre où ma femme était allongée. J'ai prié pour elle mais je ne savais plus quoi promettre à Dieu contre sa vie, j'avais déjà depuis longtemps proposé ma propre vie, pourtant vu qu'il semblait que ce soit peu de chose, j'ai encore médité un moment puis je suis allé au pied de la montagne, où j'ai monté la garde. Le deuxième matin, j'ai attendu devant sa porte qu'elle s'éveille et quand j'ai entendu qu'elle chantait à voix basse sa chanson préférée, je suis entré et j'ai vu qu'elle était guérie. Ses cheveux brillaient, son teint était rosé comme auparavant, quand je l'ai surprise en venant silencieusement l'embrasser et l'enlacer, ses yeux avaient retrouvé leur éclat, beaucoup trop audacieux et, comme auparavant, avec une pointe de vanité, grâce à Dieu, sa force était invincible. J’ai payé les guérisseurs et libéré leur famille. Ils se sont courbés jusqu'au sol car, apparemment, ils n'avaient jamais vu autant d'argent en une seule fois, ils étaient donc encore plus miséreux que nous. Après quelques heures, ils sont revenus vers moi et ont voulu me rendre une partie de l'argent. Je leur ai donc dit de donner l'argent aux pauvres de la région, cela les a totalement apaisé et ils sont partis d'un pas plus léger.

9 J'ai encore attendu quelques jours dans la région avec mes hommes et ma marchandise, le temps d'être totalement sûr que ma femme soit guérie, ensuite nous avons quitté le village par le pont pour rejoindre un des chemins, qui mènent vers le sud en longeant le col le plus proche. Alors qu'on était en train de traverser le pont, nos chevaux ont commencé à s'effrayer. Nous étions partis à l'aube et il n'y avait personne à la ronde sauf le fils de l'aubergiste que nous venions d'engager comme guide pour qu'il nous conduise jusqu'au col. Sur le pont, les chevaux ont commencé à se cabrer comme des furibonds, et quand j'ai vu que le cheval rouan de mon épouse s'effrayait et qu'elle risquait de le lâcher, je suis descendu de mon cheval et suis parvenu à la retenir dans la cohue de mes hommes, des animaux et des marchandises. Alors qu'elle me regardait avec reconnaissance, la croupe d'un autre cheval m'a fait basculer du pont dans l'eau. J'ai eu de la chance, s'il m'avait donné un coup, je ne serais plus vivant. Sous le pont, la rivière était peu profonde, je suis remonté, me suis mis en selle et nous avons repris notre chemin. Après quelques heures, j'étais sec. Toutefois, le changement de comportement de notre guide n'a pas pu m'échapper. Quand il a vu que j'étais tombé dans la rivière, des marques d'épouvantes ont commencé à poindre sur son visage. Je suis en selle à côté de toi, l'ami, lui ai-je dit, il ne m'est rien arrivé, j'ai seulement un peu barboté dans votre eau aux propriétés curatives. Au plus il avait l'air désolé, au plus je riais. L'expression de son visage relevait même les commissures des lèvres de ma femme. Il m'a demandé si j'étais tombé en entier dans l'eau. J'ai dit que oui. Ensuite, il m'a demandé si j'avais immergé tout mon corps et ma tête. J'ai encore répondu que oui. «Alors que soit avec toi la clémence de Dieu » dit-il. «Pourquoi ? » ai-je demandé. "Les eaux de quatre ruisseaux confluent dans la rivière » m'a-t-il répondu. «Les savants disent que cette petite rivière est la dernière survivance du paradis qui est, d'après les livres saints, situé dans notre province ici à la frontière avec l'Arménie. Et c'est là qu'il prend sa source. ", le fils de l'aubergiste a ensuite ajouté 
avec une voix désespérée «que l'eau de cette rivière rendait immortel. A cause de la corruption du monde, son pouvoir a déjà commencé à s'affaiblir, mais si tu y plonges tout ton corps, alors on dit que tu n'échapperas pas à l'immortalité. » J'ai corrigé sa phrase en lui disant que l'homme tente d'échapper à l'épée de son ennemi, à une maladie grave ou à une balle tirée d'une embuscade, mais pas à l'immortalité. « Dieu, prends pitié de lui car il ne connaît pas la puissance de tes actes » a ajouté notre guide et ses yeux baissés n'ont ensuite regardé que la poussière du chemin et les premières fleurs printanières qui avaient percé dans la neige fondante. «Ce sont des superstitions » a dit mon frère, « des superstitions d'attardés qui ne savent même pas qu'autour la guerre gronde, que le pouvoir change et qui ont seulement leur vielle pensée inculte. " Mon frère a ri et encore heureux qu'il n'est pas tombé de cheval. Des superstitions... j'ai ri avec lui. Après avoir atteint le col et payé le garde, nous avons traversé la frontière, à présent, nous étions déjà en Perse, le chemin vers Tabriz était ouvert.

10 A Tabriz, j'ai acheté une maison, ma femme a mis au monde quelques enfants, j'ai lancé une affaire et, avec la complicité de mon frère, j'ai fait du commerce à travers toute la Perse, la Turquie et l'Arménie. Je pense que la chance avait commencé à sourire à ma famille. Un jour, dans les faubourgs de Bagdad, nous ne sommes pas parvenus à convenir avec un autre négociant du prix du curcuma, et il a commencé à nous servir ses critiques. A la fin, il a dit: "C'est ton aîné qui devrait tenir le commerce, pas toi. » Je lui ai ri au visage, pourtant le lendemain je me suis regardé dans un des miroirs que l'on vendait sur le marché. J'ai été pétrifié, mon frère commençait à avoir des cheveux gris, moi pas. La peau de mon frère était chaque jour couverte par de nouvelles rides, la mienne était comme avant. Ce jour-là, tout effaré, j'ai chevauché mon cheval et l'ai fait crever, tout comme d'autres, avant d'arriver à la maison. Quand j'ai ouvert la porte, j'ai heurté un serviteur et j'ai couru à bout de force et le cœur palpitant dans la chambre de ma femme, je l'ai prise dans mes bras, emportée sur le balcon, et, sous le soleil de midi, j'ai commencé à compter ses rides. Tout d'abord, elle a ri, ensuite elle a balbutié et, tout comme les domestiques, elle n'a pas saisi le sens de mon comportement. Depuis notre dernier voyage, depuis sa maladie, et après les naissances de mes enfants, elle avait commencé à avoir des rides. Elle avait des rides qui n'existaient pas il y a un an, elle avait des rides causées par le rire et les soucis, elle avait des rides causées par l'étonnement, la surprise et l'assurance des prières, elle avait des rides à cause de ses lèvres serrées pendant l'amour et à cause des ragots licencieux des discussions entre femmes. Ensuite, j'ai pris le plus grand miroir que nous avions à la maison et l'ai orienté vers mon visage. A nouveau, j'ai été pétrifié, je me suis observé comme si j'étais ensorcelé, puis je l'ai brisé. Mon visage n'avait pas changé au cours de ces quelques derniers automnes. Les cicatrices que je m'étais faites étaient totalement effacées et je ne pouvais même plus indiquer leur place. Il n'y avait aucune ride sur mon visage, il était toujours le même. C'est alors que j'ai compris ce qui m'arrivait, j'étais devenu immortel, j'étais maudit. J'aillais voir mourir les personnes qui me sont chères, j'allais voir mourir ma femme adorée, j'allais la voir devenir vieille, j'allais être démuni de la fatigue de ma propre vieillesse à ses côtés, j'allais voir comment mon frère, mes enfants, mes amis et même mes ennemis deviennent une file d'ombres emmenée par Azraël, moi seul allais rester le même, inchangé, maudit. Que Dieu, dont la majesté est sans fin, me pardonne mon orgueil et consente à ce que je lave mes pêchers dans la souffrance. Que Dieu, qui est éternel, m'accorde d'atteindre le terme de mon temps. 
11 Une nuit alors que je ne parvenais pas à trouver le sommeil, dans un accès de chagrin et l'âme pleine de lamentations, j'ai confié à ma femme l'horreur de mon secret. Ce jour-là, Dieu m'a gratifié d'une nuit pénible et sans rêve. Quand j'ai ouvert les yeux le lendemain, j'ai vu que ma femme avait passé une nuit blanche, elle avait dû veiller sur mon sommeil, en chassant loin de moi les esprits des ténèbres et en appelant un bataillon d'anges pour nous aider dans notre triste destin. Le lendemain, encore avant l'aube, elle m'a pris par la main et m'a conduit chez son oncle, le frère aîné de son père, qui habite dans les faubourgs. Nous lui avons raconté notre histoire. Tout en buvant du thé, il nous a écouté avec attention, alors que ses petits yeux se perdaient dans les crevasses de sa peau ratatinée. Ses mouvements étaient doux, ses rides profondes et sa peau me rappelaient la peau d'un éléphant africain. Quand nous avons terminé notre récit, il m’a regardé sombrement et m'a dit: "Mon fils, tu as trop souvent offensé Azraël, on dirait que ton arrogance ne connaît pas de limites. » Après une telle offense, je me suis redressé et seule ma considération devant son âge avancé m'a retenu d'aboyer quelques jurons sur son compte dont la comparaison entre son âme et celle d'un chien dégénéré et galeux aurait été la plus douce. Le vieillard m'a regardé de biais et a commencé à rire en silence. Ensuite, il m'a appelé pour que je lui donne quelques livres et quelques rouleaux de papier posés sur une étagère qu'il ne parvenait plus, comme il le disait lui-même, à atteindre depuis longtemps et dont il n'avait plus eu besoin depuis des années. Il a ouvert les livres, déroulé les rouleaux de papier longuement rédigés et les parchemins dégoulinants de lettres étrangères, il a commencé à les lire et a cessé de s'intéresser à nous.

Ce matin-là, le soleil venait juste de se lever et mon épouse a souri pour la première fois, elle m'a dit qu'il avait toujours été comme ça, qu'elle se rappelait surtout comment il était plongé dans ses livres et comment il avait appris à préparer des pâtisseries sucrées et moelleuses pour que les enfants ne le dérangent pas. Quand les enfants étaient calmes et qu'ils ne le dérangeaient pas, ils pouvaient aller jouer en silence dans la pièce près de lui et recevaient des sucreries, s'ils n'étaient pas calmes, ils en recevaient quand même car son oncle n'avait pas le cœur de leur refuser. Il n'a jamais tenté de se marier, quelle femme du reste aurait voulu d'un pareil pauvre qui ne vit que pour ses livres et seulement de l'aumône et du respect des autres? Tous les enfants des environs l'aimaient pourtant bien. Après être resté assis plusieurs heures dans son pauvre logis, après avoir amené de l'eau, après avoir caché et chassé les pensées désagréables comme on le ferait avec des moustiques, vers le soir, alors que je venais juste de décider d'allumer la lampe, l'oncle s'est soudainement levé et a fermé un de ses livres. Il m'a regardé et a dit: "Ton problème est plus intuitif que reconnu et décrit par les savants, cependant je vais t'expliquer ce qui s'est passé. D’après les écritures saintes, le paradis se situe en effet originellement en Arménie. Tout ce que les paysans t'ont dit est a peu près la vérité. Comme tu le sais peut-être, notre religion, la religion des barbares et même la religion des chiens chrétiens rêvent chacune du paradis. Là, notre âme sera délivrée de la grotte des ténèbres et de l'ignorance, là nous voudrons rencontrer Dieu, dont la gloire durera pour toujours. Même cette religion étrange et millénaire, qui ne connait pas Dieu et dont le fondateur a été apparemment touché par une étincelle divine dans une forêt de cerfs quelque part en Inde, a son sanctuaire dans la région dont tu m'as parlé. Il ne m'avait jamais semblé auparavant qu'il puisse y avoir quelque chose de vrai dans ces légendes. » Et ensuite, l'oncle de ma femme a commencé à parler des différentes sortes d'anges, de la porte qui conduit d'un ciel à l'autre. Du paradis auquel a accès seulement celui qui est convenablement préparé. Il m'a montré nos écritures saintes et celles des païens et des 
barbares. Il voyait un rapport entre ce qui m'était arrivé et les vers de certains prophètes, et pas seulement nos prophètes mais aussi ceux des barbares, il trouvait des relations et des contradictions qui, à nouveau, entre des choses pourtant inaccordables, se transformaient, dans l'harmonie de Dieu, et, d'après mon opinion misérable, inculte et malingre, étaient comme une fleur oubliée en train de sécher sur le marché, la première rencontre des amants ou la révélation des saints.

J'ai tout écouté avec attention, j'étais stupéfait et horrifié par ses visions et son érudition, mais je n'ai pas du tout compris comment tout cela concernait mon destin en piteux état, je ne savais pas où mon kismet se trouvait dans tout cela. Mon esprit s'est affaibli et mon désespoir a pris appui sur mon dos voûté. A ce moment, ma femme est entrée dans la bibliothèque avec le souper. Son oncle se tenait le dos tourné et, le regard fixé sur le jardin, il m'a expliqué quelque chose sur l'ange Raziel qui est le plus proche serviteur de Dieu et qu'un simple paravent sépare de son maitre. Sans qu'il n'ait bougé la tête et sans que ma femme n'ait parlé, il lui a répondu: "Mais naturellement, ma petite, je sais, pardonne à ton vieil oncle qui seulement s'enivre avec présomption de ses paroles. Un couteau. » dit-il en se tournant vers moi : « qu'est-ce que tu comprends le mieux, dis-moi ce que tu comprends le mieux et j'essayerai de te conseiller, mon petit. » Je ne savais pas quoi dire. J'ai voulu dire que je m'y entendais en commerce, il a acquiescé avant que je n'ouvre la bouche, j'ai voulu dire qu'au cours de ces dernières années, les meilleurs modèles récents de fusils ont été écoulés, avant même que je ne commence à parler, il a secoué la tête avec mécontentement. Après quoi, $\mathrm{j}$ 'ai dit: «Je suis de loin le meilleur marchand d'épices. J'achète et je vends de l'anis, de la badiane, de l'estragon, du galanga, de la cardamome, des feuilles de laurier, du curcuma et de la coriandre, de la fleur de muscade et des noix, je vends du sumac et du safran, du gingembre et de l'hysope. J'aime l'odeur des épices tout comme j'aime le tintement de l'argent que l'on me donne.» "Qu'arriverait-il, m'a-t-il demandé, si tu mélangeais du piment piquant, du poivre blanc, un peu de fenugrec, du curcuma, de la coriandre et de la cardamome?» J'ai éclaté de rire : «Tout le monde sait que c'est la base du mélange d'épices pour le curry. », ai-je dit. "Oui », opina-t-il avant d'ajouter : "Ton caractère a trop de piment piquant et trop de poivre colérique, tu es de nature impulsive et, dans ta vie, tu as plusieurs fois croisé le chemin d'Azraël, l'ange de la mort, et apparemment tu l'as aussi rencontré non loin de son domaine, non loin de la région qui est une survivance du paradis. N'as-tu pas eu de remords lorsque tu as tué le serviteur qui voulait te voler?", m'a-t-il demandé. Sa question était claire, ma réponse aussi. "Bien sûr que non, me suis-je écrié, comment sinon aurais-je pu faire du commerce, comment sinon aurais-je réussi à tout contrôler, comment sinon aurais-je entretenu ma famille? Comment?» Le vieillard a regardé ma femme et haussé les épaules, puis il dit : «Beaucoup trop de feu, ma petite, beaucoup trop de feu. Quand vous êtes descendu dans la vallée par le chemin qui rappelle une pelote de vipères et qui est, certainement à ton plus grand étonnement, décrit dans un des livres que j'ai acheté à la Médina, a continué le vieillard, vous avez dépassé l'ancienne frontière de l'Eden, région dans laquelle était autrefois situé le paradis, et là tu as tué ton serviteur, ce qui a causé la damnation que tu dois maintenant supporter. L'ange de la mort s'est ensuite seulement vengé en te laissant faire trempette dans l'eau de l'immortalité. Azraël a, en tout cela, fait preuve d'une ingéniosité certaine, on pourrait même dire, d'ironie. ", a-t-il dit puis il s'est tu. Je n'ai pas compris de quoi il parlait, mais cette fois je me suis décidé à ne pas l'interrompre. «La seule chance, a-t-il continué, la seule chance qu'il te reste à présent est l'expiation. » "Qu'est-ce que cela signifie?», ai-je demandé. «Cela signifie que, déjà, tu ne prendras plus jamais la vie d'un autre homme, cela signifie que tu 
mettras ton impulsivité au service de ceux qui sont impuissants et que Dieu a doté de souffrance. " "Tu veux peut-être que je devienne un mendiant et un nécessiteux ? », lui ai-je demandé en haussant le ton. « Tant que tu resteras contraire, je ne t'aiderai pas. »at-il répondu. " Aide-le, petite, s'il n'est pas déjà trop tard. », a-t-il dit à ma femme et il a pris une telle expression qu'il était clair que nous devions partir. Après quelques jours, j'ai envoyé mon frère chez le vieillard pour savoir ce que tout cela signifiait, ce que je devais faire, pour qu'il m'explique tout, purement et simplement, je ne suis qu'un commerçant ordinaire, pas un savant comme lui. La réponse a tinté : "Défais-toi de toutes les choses que tu aimes le plus. » Après plusieurs jours d'accablement, de colère et d'intense furie, j'ai offert le meilleur et le plus fougueux de mes étalons au gérant de l'orphelinat municipal pour qu'il le vende et ait ensuite de l'argent pour donner des vêtements et de la nourriture aux enfants. Il a vendu à grande perte mon meilleur cheval au marché ; lorsque je l'ai appris, un tel accès de rage m'a envahi que je me suis aussitôt précipité chez lui. Quand je suis arrivé dans le local où il était courbé sur son livre de comptes désespérés, et que j'ai voulu le sermonner parce qu'il avait tiré moins du tiers de la valeur réelle du pur-sang, j'ai été interrompu par deux enfants, un garçon qui était en train de s'enfuir avec le foulard de sa sœur. Je ne sais pas pourquoi mais j'ai eu l'impression que je connaissais ces deux enfants de quelque part. Au début, la fille suppliait son frère et, à la fin, elle a essayé de le mater. Le gamin était pourtant plus grand d'au moins une tête, se hissait sur ses pointes et tenait le foulard en levant les bras au dessus de sa tête. Sa sœur ne parvenait pas à l'atteindre. Le gérant de l'orphelinat a grondé le garçonnet, lui a pris le foulard délavé, l'a rendu à sa sœur, les a poussés vers la cour et m'a demandé: "Tu ne connais pas ces enfants? Tu ne les reconnais pas? Il y a quelques années, leur plus grand frère a pris part à une de tes expéditions marchandes. On raconte qu'il est mort quelque part dans les montagnes. Ils n'avaient personnes d'autres, leurs parents étaient morts depuis longtemps et il ne leur restait que leur grand frère qui essayait de subvenir à leur besoin, mais vu qu'il est mort au cours d'une de tes expéditions dans la montagne, ils sont devenus des orphelins. » J'avais la tête qui tournait et, lorsque je suis tombé sur le banc, j'ai vu que le gérant me tenait par les épaules. Ces enfants étaient le frère et la sœur de mon serviteur, du serviteur qui avait voulu me voler et que j'avais froidement tué. Le gérant m'a parlé encore un moment mais je n'entendais pas ce qu'il disait, je voyais seulement qu'il ouvrait la bouche et que ses yeux avaient l'air perdu. Je ne me suis remis qu'après qu'il m'ait mis une tasse de thé en bouche. Je suis parti et, le lendemain, je lui ai donné de l'argent pour qu'il agrandisse son institution. Après plusieurs jours, je suis retourné voir le gérant et je l'ai interrogé en détail sur la possibilité d'adopter le frère et la sœur de l'homme que j'avais tué, il s'est réjoui et m'a dit que tout ce qu'il avait entendu sur moi était faux et que ce n'était pas vrai que mon âme était dure comme la pierre. Lorsque que j'ai vu que l'on pouvait lire sur son visage comme dans un miroir, que l'on pouvait voir sur sa figure chaque sursaut de son âme, j'ai été étonné qu'il n'ait pas vendu mon pur-sang pour encore moins d'argent. Ensuite, ces enfants sont administrativement devenus les miens et, vu qu'ils étaient plus âgés que ceux mis au monde par ma femme, ils allaient donc, d'après la loi, recevoir la plus grande part de mes biens si Azraël revenait sur sa décision et me laissait mourir un jour. Par la suite, ma femme m'a dit qu'en dormant, j'avais crié plusieurs nuits comme elle ne m'avait même jamais entendu parler, j'aurais, d'après elle, crié des mots comme faute et honte, j'aurais imploré le pardon. Je me souviens encore aujourd'hui de certains de ces rêves, ils étaient durs comme la justice de Dieu dont la sollicitude nous écrase autant que lorsque nous broyons des olives dans une presse en bois pour en extraire l'huile. Quelques mois 
plus tard, j'ai entendu l'un de mes commis dans un magasin de tapis dire à un autre : « Notre monsieur a bien décliné en quelques semaines, il a soudain vieilli de manière inattendue.» Ils n'ont jamais su pourquoi je leur avais donné une semaine de congé et pourquoi j'avais payé à l'un d'eux la dote de sa fille.

Ce jour-là, je me suis rendu chez l'oncle de ma femme. La maison était abandonnée et le vieux était parti. J'ai appris par le voisin qu'il était parti acheter quelques manuscrits sacrés quelque part près de la frontière arménienne. Lorsque je l'ai dit à ma femme, elle m'a dit qu'elle était allée de temps en temps chez lui pour cuisiner ou nettoyer et qu'il nous avait laissé une lettre chez le voisin. Je n'en ai jamais vu depuis cette époque une lettre aussi somptueusement calligraphiée. L'oncle y souhaitait une bonne vie pour moi et pour mon épouse, qu'il continuait d'appeler ma petite, et il y disait que le destin de ma vie était retourné dans le lit ordinaire de la rivière. Et ensuite, à la manière des vieilles personnes, il se remémorait longuement les jeux de ma femme avec d'autres enfants dans sa pièce au temps de sa jeunesse. Au temps où il était encore plein d'orgueil et d'insolence pour pouvoir tirer le rideau dissimulant les intentions de Dieu. Maintenant toutefois, il avait soi-disant compris que notre destin avait aussi été pour lui une invitation à avancer dans l'inconnu.

Dernièrement, je me suis surpris à me souvenir de ma jeunesse, comme en témoignent mes récits, et seuls les vieux et les personnes malades font cela. Et c'est ce que je suis à présent, un homme vieux et malade qui attend qu'Azraël vienne le chercher. Je pressens que sa visite va correspondre avec le mûrissement des olives, et j'espère que j'aurai encore le temps de terminer la récolte de cette année. »

Tout en écoutant le récit, le docteur Lukavsky prit quelques notes et le lendemain il alla à la bibliothèque où il compara quelques tomes des récits Les mille et une nuits. D'abord, il commença par la table des matières. Ensuite, il regarda dans l'index. Finalement, il parcourut les titres des contes et en lut certains. Il ne trouva pourtant pas ce récit. Après plus d'une heure, il se rendit compte qu'il ne pouvait pas consacrer plus de temps à ses recherches car il avait des consultations dans l'après-midi. Apparemment, je l'ai passé, estima-t-il. Apparemment. Ce récit doit bien venir de quelque part, pensa Antonin, toutefois, débordé par le travail, il ne s'intéressa pas plus en détail à la source de l'imagination du confiseur.

\section{INDEX}

Mots-clés : littérature tchèque

Index géographique : République tchèque

Index chronologique : époque contemporaine, XXe siècle

oeuvretraite Zmeškal T. : Milostný Dopis Klínovým Písmem 


\section{AUTEURS}

SARAH FLOCK

Docteur en Littérature slave (Université Libre de Bruxelles, Université Charles de Prague) ;

membre du comité de rédaction de Slavica Bruxellensia 